\title{
Meta
}

Journal des traducteurs

Translators' Journal

\section{OAKes, Michael P. et Ji, Meng (dir.) (2012) : Quantitative Methods in Corpus-Based Translation Studies. A Practical Guide to Descriptive Translation Research. Amsterdam/Philadelphia : John Benjamins, 361 p.}

\section{Geneviève Has}

Volume 59, numéro 2, août 2014

URI : https://id.erudit.org/iderudit/1027488ar

DOI : https://doi.org/10.7202/1027488ar

Aller au sommaire du numéro

Éditeur(s)

Les Presses de l’Université de Montréal

ISSN

0026-0452 (imprimé)

1492-1421 (numérique)

Découvrir la revue

Citer ce compte rendu

Has, G. (2014). Compte rendu de [OAKES, MiCHAEL P. et Ji, MENG (dir.) (2012) : Ouantitative Methods in Corpus-Based Translation Studies. A Practical Guide to Descriptive Translation Research. Amsterdam/Philadelphia : John Benjamins, 361 p.] Meta, 59(2), 464-466. https://doi.org/10.7202/1027488ar d'utilisation que vous pouvez consulter en ligne. 
from Ancient Languages into Estonian: Outlines for Translation History» et la chronologie de l'histoire de la traduction poétique estonienne de Marie-Kristina Lotman «Equimetrical Verse Translation in Estonian Poetic Culture». Il ne faut pas non plus oublier le lien viscéral de la traduction avec l'ethnologie comme le précise le travail d'un Georges Devreux et d'autres travaux plus récents. La traduction va bien au-delà des sciences humaines pour englober dans sa sphère les études littéraires, comme lorsqu'elle sert de base à la littérature comparée. Anne Lange, codirectrice de l'ouvrage «Transitions in the Intercultural Locale: A Case of Two Estonian Translators", s'inspire des cas de deux traducteurs estoniens afin de se prononcer sur ce lieu de chevauchement des deux cultures dans lequel le traducteur œuvre, influencé par «la realia socio-politique» (p. 184) de la culture cible. Ce lieu spécifique, comme elle aime à dire, n'est ni la source ni la cible, ni un troisième lieu, mais une intersection qui affirme le potentiel des multiples perspectives et les «negotiating possibilities» (p. 184) pour l'interprétation du texte source. "The visibility of the translator depends on the backdrop: it has to be that of the original. Otherwise he would be the domesticating, invisible and fluent translator of Venuti» (p. 183).

Les articles suivants sont des études synchroniques de l'histoire de la traduction en Estonie. Aile Möldre, dans «Publications of Literary Translation in Estonia in 1901-1917: An Overview», s'attelle à retracer la traduction littéraire couvrant la période allant de 1901 jusqu'à 1917 pendant laquelle, depuis la révolution de 1905, l'industrie de la traduction littéraire a connu un essor considérable, de l'allemand et du russe $(80 \%)$ et du français, du finnois et des langues scandinaves. Elin Sütiste, dans «Images of Literary Translation in Estonian Translation Criticism 1906-1940», pour sa part, s'attache à la critique de la traduction littéraire depuis le début du $\mathrm{xx}^{\mathrm{e}}$ siècle jusqu'à la Deuxième Guerre mondiale. Les années du «total Stalinism» (p. 187) et le cas de l'après-guerre soviétique en Estonie sont le sujet de débat de Daniele Monticelli, le troisième codirecteur de " "Totalitarian Translation" as a Means of Forced Cultural Change: The Case of Postwar Soviet Estonia». L'objectif de la traduction totalitaire était d'éradiquer les traditions culturelles locales et de facto implanter les valeurs soviétiques. Dans ce contexte, le traducteur devient une marionnette idéologique et perd sa qualité d'agent culturel autonome. De la traduction de l'histoire est née l'histoire des littératures périphériques. C'est sous cet angle que Katiliina Gielen, dans "Authors as Translators: Emerging Hierarchical Patterns of Literary Activity in Early Soviet Estonia », retrace l'époque staliniste (1944-1956) en vue de cerner les modèles hiérarchiques de l'activité littéraire au début de l'Estonie soviétique.

Point n'est besoin d'insister sur le lien cornélien entre la traduction et la sémiotique; Ülar Ploom, dans "From Mugandus [Accommodation] towards Discourse-Aware Translation", s'appuie sur la théorie du polysystème de manière à périodiser l'itinéraire italien dans l'histoire de la traduction en Estonie. Il insiste sur le fait de distinguer les «faits» et les traductions «valeurs». Pour finir, Ene-Reet Soovik, dans «Estonian Literary Translation in the Early 21st Century: On the Context and the Content», fait l'inventaire des fictions littéraires traduites depuis le début du $\mathrm{XIX}^{\mathrm{e}}$ siècle. C'est ainsi que la boucle est bouclée et que cet exercice de périodisation prend fin. Cette partie suit un cheminement logique qui part des premières lueurs de systématisation pour aboutir aux dernières parutions en histoire de la traduction en Estonie.

En dépit de quelques redites inéluctables de l'histoire et de la théorie de la traduction, les études de cas retenues nous offrent un aperçu contenant moult références et une bibliographie riche et diversifiée. Pour finir, nous dirons que le mot-clé de cette compilation reste l'interdisciplinarité de la traduction: le spectre de la traduction est large, nous ne cesserons de le répéter! Grâce à cet effort conjugué, de part et d'autre, nous espérons que l'Estonie, pays né de et dans la traduction comme le souligne Meschonnic (p. 9), trouvera des éléments de réponse aux nombreuses lacunes de son histoire de la traduction.

Hanane Belgaid Université Ibn Tofaill, Kénitra, Maroc

\section{RÉFÉRENCE}

NidA, Eugene A. et TABER, Charles R. (1969): The Theory and Practice of Translation. Helps for translators, vol. 8. Leiden: E.J. Brill.

Oakes, Michael P. et Ji, Meng (dir.) (2012): Quantitative Methods in Corpus-Based Translation Studies. A Practical Guide to Descriptive Translation Research. Amsterdam/Philadelphia: John Benjamins, 361 p.

Quantitative Methods in Corpus-Based Translation Studies est le $51^{\mathrm{e}}$ volume de la série Studies in Corpus Linguistics de John Benjamins et, il faut le souligner, le premier ouvrage de cette série à être entièrement consacré à la traductologie. Quelques chapitres des volumes précédents se sont tout de même aventurés sur ce terrain, notamment celui de Sara Laviosa dans Perspectives on Corpus Linguistics en 2011, mais sans pouvoir (ni vouloir 
pouvons-nous évidemment supposer) prétendre à l'exhaustivité du présent ouvrage. Oakes et Ji remarquent avec justesse en préface qu'il n'existe aucune "systematic description of the various statistical tests that may be adapted from corpus linguistics in general for the purpose of translation research» (p. VII). De fait, poursuivent-ils, malgré l'intérêt grandissant que les chercheurs en traductologie manifestent pour les méthodes à base de corpus, plusieurs des études qui utilisent ces dernières sont restées exploratoires et cette lacune ne peut que ralentir les progrès de la discipline (p. VII).

Ainsi, destiné aux étudiants et chercheurs, cet ouvrage se veut un guide détaillé, pour l'application en traductologie de méthodes quantitatives propres à la recherche à base de corpus. Il s'agit donc de rendre digestibles ces méthodes à un public curieux et non spécialiste. Le pari est ambitieux, mais réussi, croyons-nous. En effet, les directeurs de ce volume proposent à leurs lecteurs un ensemble bien dosé d'outils statistiques de base, de démonstrations avancées et d'études de cas éclairantes. Qui plus est, si le contenu peut paraître rébarbatif au premier regard, une lecture plus attentive corrige rapidement ce pronostic et chacun y trouve son compte.

L'ouvrage est divisé en quatre parties: «Theoretical exploration", "Essential corpus statistics", "Quantitative exploration of literary translation" et «Quantitative exploration of translation lexis». La première partie, comme son nom l'indique, s'emploie à tisser des liens entre les théories de la traduction et les méthodes qualitatives et quantitatives de la recherche à base de corpus. La deuxième partie présente des études de cas qui mettent en lumière les outils statistiques permettant de mesurer et de décrire les corpus de traduction. La troisième partie, quant à elle, traite de l'application de méthodes quantitatives à des corpus de traduction littéraire. Enfin, la quatrième partie s'aventure sur le terrain de la lexicométrie particulière aux textes traduits.

Le coup d'envoi est donné par Barbara Lewandowska-Tomaszczyk, qui se propose d'observer les traces que laissent les différents cycles de «reconceptualisation" caractéristiques de la traduction à l'aide de méthodes quantitatives et qualitatives choisies. S'appuyant sur un corpus anglais et polonais (p. 5), l'auteure présente une analyse détaillée qui met en évidence une "translational asymmetry» (p. 29) engendrée par la tendance des concepts à changer de sens en fonction des domaines d'une langue donnée. En d'autres termes, Lewandowska-Tomaszczyk avance que l'équivalence gagnerait à être appliquée à des domaines conceptuels plus larges plutôt qu'à des unités linguistiques individuelles (p. 30).
Le chapitre suivant présente un aperçu de l'utilisation de techniques de régression logistique et linéaire binomiales à l'aide du logiciel de programmation R. La démonstration s'appuie sur le corpus INTERSECT, qui regroupe des textes variés en anglais et leurs traductions en français et en allemand. Stefan Th. Gries et Stefanie Wulff offrent un excellent travail de vulgarisation et arrivent à piquer la curiosité du lecteur sans connaissances préalables en sciences statistiques. Les auteurs terminent leur présentation en remarquant avec justesse que ces techniques sont particulièrement fiables lorsqu'il s'agit de débusquer des motifs ou des récurrences autrement invisibles, même à l'œil entraîné.

Meng Ji clôt la première partie de l'ouvrage avec une étude stylistique comparée de Don Quijote de La Mancha et de deux traductions chinoises du $\mathrm{Xx}^{\mathrm{e}}$ siècle. Ji cherche à montrer à la fois les possibilités et la richesse méthodologique d'une approche qui combine l'analyse qualitative et quantitative de traductions (p. 55). Tout en détaillant sa méthode, Ji souligne ainsi les relations de dépendance qu'entretiennent les deux traductions chinoises entre elles, mais aussi avec l'original, par delà «the huge linguistic and social and cultural differences » qui caractérisent ces textes (p. 70).

L'étude suivante montre les défis que pose la création de corpus de traduction et propose des pistes techniques pour la conversion de textes (p. 76). Se basant sur leur expérience avec le Norwegian Spanish Parallel Corpus, Lidun Hareide et Knut Hofland offrent un survol concis, mais riche des particularités et difficultés de la conception de corpus pour les besoins de la traductologie, de la sélection des textes à l'alignement, et finissent avec quelques outils permettant de valider le potentiel descriptif du corpus.

Michael P. Oakes poursuit, dans le même ordre d'idées, avec une vue d'ensemble des techniques statistiques servant à décrire les corpus de traduction. Le chapitre, très technique, réussit non seulement à vulgariser efficacement des méthodes statistiques particulièrement arides, mais aussi à montrer de quelle façon elles peuvent être appliquées et les résultats que l'on peut en espérer.

En contrepoint, Shih-Wen Ke se propose d'explorer des méthodes de regroupement pour l'analyse des données tirées d'un corpus de traduction, notamment l'approche hiérarchique et l'approche de partition. Ces dernières sont minutieusement expliquées, illustrées et comparées alors que Ke prend soin de préciser quelle méthode est à privilégier en vue d'un objectif particulier, puisque "Cluster analysis is a series of carefully assembled tasks with each component task having the potential to change the outcome of the analysis» (p. 169). Cette mise en garde ne devrait 
toutefois pas décourager l'emploi des méthodes de regroupement, mais vise en fait à fournir toute l'information nécessaire à leur utilisation.

La rubrique suivante consiste en une étude comparée de deux traductions partielles vers l'anglais de Hongloumeng, de Cao Xuequin. Ji et Oakes profitent de l'occasion pour présenter «a set of statistical tests for corpus-oriented Translation Studies» (p. 176). La section propose donc, en contexte d'utilisation, des outils comme le test de Student, le test $\chi^{2}$ (chi-squared) ou le coefficient de corrélation linéaire de Bravais-Pearson, entre autres. Les auteurs soulignent de ce fait le rôle fondamental que peuvent jouer les méthodes statistiques en vue de consolider et élargir l'approche empirique en traductologie.

Jon M. Patton et de Fazli Can proposent ensuite une étude stylométrique d'une traduction vers le turc de Dubliners, de Joyce. Il s'agit ici d'identifier, à l'aide de marqueurs spécifiques, les caractéristiques stylistiques de l'original conservées dans la traduction. Les possibilités ouvertes par les auteurs en conclusion dépassent le cadre de la traductologie et vont jusqu'à la lutte contre le plagiat, notamment.

La deuxième partie de l'ouvrage se termine avec la contribution de Jan Ribicki, consacrée elle aussi à la stylométrie. Cette fois-ci, par contre, il est question de la présence, visible ou non, du traducteur dans les corpus traduits. Alors que traditionnellement la méthode Delta sert à déterminer l'auteur d'une œuvre, Ribicki se demande s'il est plutôt possible d'en identifier le traducteur. Nous laisserons au lecteur le soin de découvrir ces résultats, mais cela nous semble une application forte de beaucoup de potentiel pour la traductologie si d'autres études pouvaient les reproduire.

La dernière section du volume est entamée par Meng Ji, qui se penche sur la terminologie scientifique, ou plus précisément sur la façon dont le vocabulaire scientifique chinois s'est enrichi à l'aide de traductions de l'anglais, du néerlandais, du japonais et du français. Ji examine deux fonctions linguistiques en particulier: la variation en longueur des unités lexicales et la création ainsi que l'utilisation de particules grammaticales fonctionnelles. Son analyse montre une partie du processus complexe qui a mené à la transformation de la terminologie scientifique chinoise par une médiation culturelle innovante.
La démonstration suivante s'emploie à expliquer les variations lexicales relevées dans un corpus de traductions du Rgveda à l'aide de méthodes de regroupement et de techniques statistiques en vue d'expliciter comment les traducteurs effectuent leur choix lorsque les textes auxquels ils sont confrontés sont ambigus.

Nous passons ensuite à une étude qui se propose de tester trois méthodes statistiques pour mesurer à la fois l'utilité et l'usage des affixes de dérivation dans l'anglais de traduction. À partir du Translation English Corpus de Mona Baker, les auteurs présentent trois techniques: l'analyse factorielle, l'analyse en composantes principales et l'analyse factorielle des correspondances. Ces trois approches, qui relèvent de l'analyse multivariée, ont chacune des avantages et des inconvénients pour étudier les corpus traduits, ce que les auteurs ne manquent pas de détailler.

Enfin, les auteurs du dernier chapitre se demandent si l'hypothèse qui veut que les textes traduits soient essentiellement différents des textes non traduits de la même langue, sans égards à la langue source. Pour tester cette affirmation, ils appliquent une méthodologie en deux étapes: d'abord une analyse des correspondances basées sur profil, en vue de créer une représentation visuelle des données, puis une régression linéaire binomiale de façon à évaluer l'impact des caractéristiques des textes (non traduits, traduits et depuis quelle langue).

En définitive, le seul reproche que nous pourrions faire aux éditeurs, et encore, a trait à l'ordre des chapitres. En effet, il nous a semblé incongru de voir certains concepts techniques laissés sans explications en début de volume, puis de les voir détaillés dans une intention clairement pédagogique plus loin. Cela dit, si le lecteur est prêt à papillonner en avant et en arrière, ce petit défaut ne diminue en rien la qualité d'un ouvrage somme toute extrêmement complet.

Geneviève Has Université Laval, Québec, Canada

\section{RÉFÉRENCE}

Laviosa, Sara (2011): Corpus Linguistics and Translation Studies. In: Vander Viana, Sonia Zyngier et Geoff Barnbrook (dir.), Perspectives on corpus Linguistics. 48. Amsterdam/ Philadelphie: John Benjamins, 131-154. 